Esther Kahana

Nelly Zilber

Joseph H. Abramson

Victor Biton

Yaffa Leibowitz

Oded Abramsky

\section{Multiple sclerosis: genetic versus environmental aetiology: epidemiology in Israel updated}

J Neurol (1994) $241: 341-346$
E. Kahana $\cdot$ N. Zilber $\cdot$ Y. Leibowitz Neuroepidemiology Unit,

Hadassah-Hebrew University Hospital, Jerusalem, Israel

V. Biton - O. Abramsky Department of Neurology,

Hadassah-Hebrew University Hospital, Jerusalem, Israel

J. H. Abramson

Department of Social Medicine,

Hebrew University-Hadassah School of Public Health and Community Medicine, Jerusalem, Israel

\section{N. Zilber}

PIREN, CNRS, Paris, France and Centre de Recherche Français de Jerusalem, Jerusalem, Israel

\section{E. Kahana (ख)}

Neurology Barzilai Medical Centre, affiliated to the Faculty of Health Sciences, Ben Gurion University of the Negev, Ashkelon 78306, Israel
Due to and unfortunate oversight, a number of proof corrections were not carried out during the final printing process:

The first sentence of the second paragraph in the introduction (p.341) should read:

The big wave of Jewish immigration to Israel after the Second World War and Israel's declaration of independence (1945-1950) consisted of immigrants from Europe, where MS is frequent and North Africa and the Middle East, where MS is relatively rare.

The fourth sentence of the Material and methods section (p.342) should read:

In the present study, an additional source of information was the Israel MS Society (after reviewing the med- ical files of their members) and the Neuroimmunological Laboratory of Hadassah Hospital, to whom cerebrospinal (CSF) was sent for oligoclonal immunoglobulin assay.

The third paragraph in the discussion (p. 345) should read: The prevalence of MS in EA immigrants to Israel was found to be three times higher than that in immigrants from AA [26]. ... In the present study we found that the prevalence and incidence of MS were significantly higher in the offspring of AA immigrants than in immigrants from these countries.

Reference 25 was incomplete:

25. Leibowitz U, Kahana E, Alter M (1969) Multiple sclerosis in immigrant and native populations of Israel. Lancet II : 1323-1325 